\title{
Higgs Boson Production in Association with a Jet at Next-to-Next-to-Leading Order
}

\author{
Radja Boughezal, ${ }^{1, *}$ Fabrizio Caola, ${ }^{2, \dagger}$ Kirill Melnikov, ${ }^{3, \ddagger}$ Frank Petriello, ${ }^{1,4, \S}$ and Markus Schulze ${ }^{2, \|}$ \\ ${ }^{1}$ High Energy Physics Division, Argonne National Laboratory, Argonne, Illinois 60439, USA \\ ${ }^{2}$ CERN Theory Division, CH-1211 Geneva 23, Switzerland \\ ${ }^{3}$ Institute for Theoretical Particle Physics, KIT, Karlsruhe D-76128, Germany \\ ${ }^{4}$ Department of Physics and Astronomy, Northwestern University, Evanston, Illinois 60208, USA \\ (Received 24 May 2015; revised manuscript received 29 June 2015; published 21 August 2015)
}

\begin{abstract}
We present precise predictions for Higgs boson production in association with a jet. We work in the Higgs effective field theory framework and compute next-to-next-to-leading order QCD corrections to the gluon-gluon and quark-gluon channels, which is sufficient for reliable LHC phenomenology. We present fully differential results as well as total cross sections for the LHC. Our next-to-next-to-leading order predictions reduce the unphysical scale dependence by more than a factor of 2 and enhance the total rate by about twenty percent compared to next-to-leading order QCD predictions. Our results demonstrate for the first time satisfactory convergence of the perturbative series.
\end{abstract}

DOI: 10.1103/PhysRevLett.115.082003

PACS numbers: $12.38 . \mathrm{Bx}, 14.80 . \mathrm{Bn}$

Further exploration of the Higgs boson discovered by the ATLAS and CMS Collaborations [1,2] will be a primary goal of the continued experimental program of the LHC. In the well-measured decay modes, $h \rightarrow \gamma \gamma, W W$, and $Z Z$, the determined couplings agree with the standard model (SM) values to $20 \%-30 \%[3,4]$. This agreement will be further probed during the upcoming LHC run. The predictions of the SM are expected to be tested to the five percent level in several production and decay modes [5], providing an exciting opportunity to discriminate between different mechanisms of electroweak symmetry breaking. In addition, new properties of the Higgs boson will be accessed through the measurement of its kinematic distributions. These measurements will test whether the tensor structures of the Higgs couplings are correctly predicted by the SM, whether additional operators mediate Higgs production and decay, and whether there are new particles that modify the loop-induced $g g H$ and $\gamma \gamma H$ couplings.

Such studies [6] are currently limited by the available statistics. However, this situation will change during Run II of the LHC, and eventually the limiting factor in the search for deviations in Higgs boson properties from predictions will be our understanding of SM theory. This is apparent from a recent coupling combination performed by ATLAS [7]. The uncertainty on the theoretical predictions dominates the systematic error budget in all of the diboson decay modes. One component of this error is the overall signal normalization, for which a precise calculation of inclusive Higgs production in the gluon-fusion channel is needed. The completion of the next-to-next-to-next-to-leading order

Published by the American Physical Society under the terms of the Creative Commons Attribution 3.0 License. Further distribution of this work must maintain attribution to the author(s) and the published article's title, journal citation, and DOI. calculation was recently announced [8]. The other major component of the theoretical error is the need for improved predictions of the differential spectra that enter every analysis. In some final states this need is obvious; for example, in the dileptonic decay of the $W W$ channel a mass peak cannot be reconstructed, and accurate calculations of both signal and background distributions are needed in order to devise appropriate experimental search strategies. Even in modes where a resonance peak can be reconstructed, such as the $\gamma \gamma$ channel, the Higgs candidate events are categorized according to their transverse momentum $\left(p_{\perp}\right)$ in order to improve the signal significance. Such a division relies upon accurate and precise theoretical predictions for the Higgs momentum $p_{\perp}$ and rapidity distributions, which are used to reweight the parton-shower Monte Carlo simulations employed by the experimental collaborations.

In this Letter, we take a major step toward improving SM predictions for several kinematic distributions employed in the analysis of Higgs boson properties, by providing a nextto-next-to-leading order (NNLO) calculation of Higgs boson production in association with a jet. Compared to previous computations $[9,10]$, we include all relevant channels and color structures. More precisely, we include the complete $g g$ and $q g$ channels, which are expected to account for most of the cross section and hence enable us to perform reliable LHC phenomenological studies. The phenomenological impact of this result spans all Higgs search channels. In the $W W$ final state it refines the division of the signal prediction into exclusive zero-jet, one-jet, and inclusive two-jet bins, and it can be used to improve the resummation of the jet-veto logarithms that accompany this division [11]. For all final states our calculation can be used to more accurately reweight the Higgs $p_{\perp}$ distribution obtained from Monte Carlo simulation. Finally, it will 
allow for the comparison of the measured differential distributions from LHC Run II with the most precise SM theory to more incisively probe the mechanism of electroweak symmetry breaking.

Our calculation also represents a technical milestone in the application of perturbative QCD to the modeling of hadronic collisions. The past few years have seen a renaissance in the development of subtraction techniques designed to turn our knowledge of the infrared structure of QCD at NNLO into actual phenomenological predictions for hadron-collider observables $[9,10,12]$. Our result demonstrates the power of these newly developed methods in assisting the continued exploration of nature at the LHC.

Our Letter is organized as follows. We first review the theoretical framework that we use to obtain the results reported in this Letter. Since this has been described in detail in our previous work on Higgs plus jet production in pure gluodynamics [9], we only sketch here the salient features of the calculation. We then present the numerical results of the computation including NNLO results for cross sections of Higgs plus jet production at various collider energies and for various values of the transverse momentum cut on the jet. We also discuss the NNLO QCD corrections to the transverse momentum distribution of the Higgs boson. Finally, we present our conclusions.

We begin by reviewing the details of the computation. Our calculation is based on the effective theory obtained by integrating out the top quark. For values of the Higgs $p_{\perp}$ below $150 \mathrm{GeV}$, this approximation is known to work to $3 \%$ or better at NLO $[13,14]$. Since the Higgs boson receives its transverse momentum by recoiling against jets, we expect that a similar accuracy of the large- $m_{t}$ approximation can be expected for observables where jet transverse momenta do not exceed $\mathcal{O}(150) \mathrm{GeV}$ as well.

The effective Lagrangian is given by

$\mathcal{L}=-\frac{1}{4} G_{\mu \nu}^{(a)} G^{(a), \mu \nu}+\sum_{i} \bar{q}_{i} \mathrm{i} \not D q_{i}-C_{1} \frac{H}{v} G_{\mu \nu}^{(a)} G^{(a), \mu \nu}$,

where $G_{\mu \nu}^{(a)}$ is the gluon field-strength tensor, $H$ is the Higgs boson field, and $q_{i}$ denotes the light quark field of flavor $i$. The flavor index runs over the values $i=u, d, s, c, b$, which are all taken to be massless. The covariant derivative $D$ contains the quark-gluon coupling. The Higgs vacuum expectation value is denoted by $v$, and $C_{1}$ is the Wilson coefficient obtained by integrating out the top quark. The calculation presented here requires $C_{1}$ through $\mathcal{O}\left(\alpha_{s}^{3}\right)$, which can be obtained from Ref. [15]. Both the Wilson coefficient and the strong coupling constant require ultraviolet renormalization; the corresponding renormalization constants can be found, e.g., in Ref. [16].

Partonic cross sections computed according to the above prescription are still not finite physical quantities. Two remaining issues must be addressed. First, contributions of final states with a different number of partons must be combined in an appropriate way to produce infrared-safe observables. This requires a definition of final states with jets. We use the anti- $k_{T}$ jet algorithm [17] to combine partons into jets. Second, initial-state collinear singularities must be absorbed into the parton distribution functions (PDFs) by means of standard $\overline{\mathrm{MS}}$ PDF renormalization. A detailed discussion of this procedure can be found in Ref. [18].

The finite cross sections for each of the partonic channels $i j$ obtained in this way have an expansion in the $\overline{\mathrm{MS}}$ strong coupling constant $\alpha_{s} \equiv \alpha_{s}(\mu)$, defined in a theory with five active flavors,

$$
\sigma_{i j}=\sigma_{i j}^{(0)}+\frac{\alpha_{s}}{2 \pi} \sigma_{i j}^{(1)}+\left(\frac{\alpha_{s}}{2 \pi}\right)^{2} \sigma_{i j}^{(2)}+\mathcal{O}\left(\alpha_{s}^{6}\right)
$$

Here, the omitted terms indicated by $\mathcal{O}\left(\alpha_{s}^{6}\right)$ include the $\alpha_{s}^{3}$ factor that is contained in the leading order cross section $\sigma_{i j}^{(0)}$. Our computation will include the $g g$ and $q g$ partonic cross sections at NNLO, $\sigma_{g g}^{(2)}$ and $\sigma_{q g}^{(2)}$, where $q$ denotes any light quark or antiquark. At NLO, it can be checked using MCFM [19] that these channels contribute over $99 \%$ of the cross section for typical jet transverse momentum cuts, $p_{\perp} \sim 30 \mathrm{GeV}$. We therefore include the partonic channels with two quarks or antiquarks in the initial state only through NLO.

In addition to the ultraviolet and collinear renormalizations described above, we need the following ingredients to determine $\sigma_{g g}^{(2)}$ and $\sigma_{q g}^{(2)}$ : the two-loop virtual corrections to the partonic channels $g g \rightarrow H g$ and $q g \rightarrow H q$, the one-loop virtual corrections to $g g \rightarrow H g g, g g \rightarrow H q \bar{q}$ and $q g \rightarrow H q g$, and the double real-emission processes $g g \rightarrow H g g g, g g \rightarrow$ $H g q \bar{q}, q g \rightarrow H q g g$, and $q g \rightarrow H q Q \bar{Q}$, where the $Q \bar{Q}$ pair in the last process can be of any flavor. The helicity amplitudes for all of these processes are available in the literature. The two-loop amplitudes were computed in Ref. [20]. The one-loop corrections to the four-parton processes are known [21] and available as a Fortran code in the MCFM program [19]. For five-parton tree-level amplitudes, we use compact results obtained using Britto-Cachazo-Feng-Witten (BCFW) recursions [22].

The difficulty in completing the NNLO calculation becomes apparent when one attempts to combine these contributions and cancel the infrared divergences that appear separately in each component. The problem is that final states with different multiplicities live in different phase spaces; this feature makes it impossible to combine them directly. The issue becomes obvious if one looks at how $1 / \epsilon$ singularities appear in different contributions. Indeed, the $1 / \epsilon$ poles coming from loop amplitudes are explicit ones, but those coming from the real-emission corrections only appear upon integration over the unresolved region of the phase space. However, since we want to keep the calculation fully differential, we want to avoid 
integrating over the phase space for higher-multiplicity processes.

To reconcile these two requirements, which at first sight appear to be mutually exclusive, we use the sectorimproved residue subtraction approach [23-26]. This is an outgrowth of the sector-decomposition method [27-29] used to compute the differential cross sections for Higgs boson and electroweak gauge bosons through NNLO. Sector decomposition uses the observation that the relevant singularities can be isolated using appropriate parametrizations of phase space and expansions in plus distributions. Sector-improved residue subtraction combines this with the idea that a prepartitioning of the final-state phase space similar to the Frixione-Kunszt-Signer subtraction used at NLO [30] allows us to extend this technique to $2 \rightarrow 2$ and more complicated scattering processes. A detailed discussion of the phase-space parametrizations needed to handle all the contributing partonic processes was given in Ref. [9], to which we refer the reader for more details. Note, however, that Ref. [9] dealt with $g g \rightarrow H+n g$ partonic processes for which both the phase space and the matrix elements are highly symmetric. For the quark-gluon channel this symmetry is lost and one has to consider a larger number of "sectors" compared to the case of pure gluodynamics.

Before discussing numerical results, we would like to point out two things in connection with the application of the sector-improved residue subtraction method. First, we note that upon applying this method, one automatically generates subtraction terms that allow extraction of $1 / \epsilon$ singularities and, at the same time, make integration of the finite remainders possible. The key point is that these subtraction terms are obtained from universal soft and collinear limits of scattering amplitudes that were computed long ago in Refs. [31-38]. The universality of these subtraction terms makes the method of improved-sector decomposition attractive and, in principle, applicable to processes of arbitrarily high multiplicity. Second, when sector-improved residue subtraction is applied to a physical process, it leads to a Laurent expansion of the various contributions to the cross section in the dimensional regularization parameter $\epsilon$; the coefficients of this expansion are computed numerically. Since final physical cross sections are independent of the regularization parameter, the quality of the $1 / \epsilon^{n}, n=4,3,2,1$, cancellation is an important check of the correctness of the implementation of the method. To show the quality of the cancellation in our case, in Fig. 1 we present various contributions to the $1 / \epsilon$ pole of the partonic cross section, together with the residual noncancellation, in the $q g$ channel. We show these quantities as functions of the distance from the partonic threshold, defined as $\beta=\sqrt{1-s_{t h} / \hat{s}}, \sqrt{s_{t h}}=\sqrt{m_{H}^{2}+p_{\perp, \mathrm{cut}}^{2}}+$ $p_{\perp, \text { cut }}$. We see that the cancellation is very good, at the level of one per mill or better. Although in Fig. 1 we display the result for the total cross section, we have also checked that

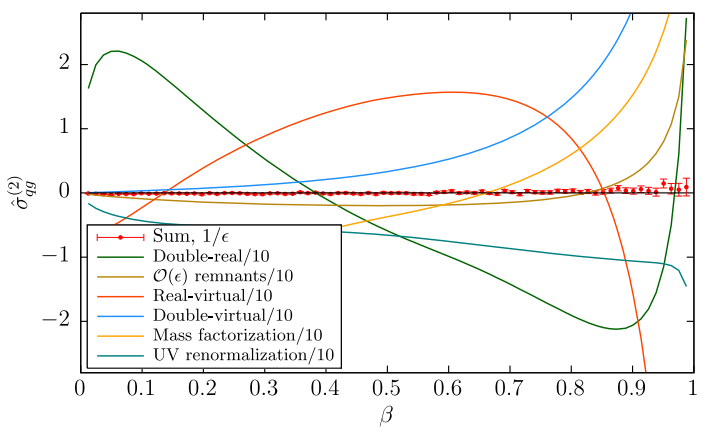

FIG. 1 (color online). Cancellation of $1 / \epsilon$ poles in the $q g$ channel. Note that individual contributions have been rescaled by a factor of 0.1 , while the sum of them is not rescaled.

the cancellation holds at a similar level for kinematic distributions. We also note that for higher $1 / \epsilon^{n}$ poles the cancellation is always better than per mill.

In addition, we have checked that our results for the Higgs plus two-jet cross section at NLO agree with MCFM [39], for both the fiducial cross section and for several kinematic distributions. We have two separate numerical implementations of the sector-improved residue subtraction method for the total cross section that agree within numerical uncertainties. Furthermore, an independent calculation was also performed using the jettiness-subtraction technique [40]. Agreement at the few per mill level for the cross section in the fiducial region $\left|y_{\text {jet }}\right|<2.5$ was found.

We now turn to the discussion of numerical results. We first compute the LO, NLO, and NNLO cross sections for inclusive Higgs plus jet production $p p \rightarrow H+j$ at the 8 and $13 \mathrm{TeV}$ LHC. We use $m_{H}=125 \mathrm{GeV}$ and $m_{t}=$ $172.5 \mathrm{GeV}$. To define the cross section, any infrared-safe jet algorithm can be used. For the results presented here, we choose the anti- $k_{T}$ algorithm with $\Delta R=0.5$ and a cut on the jet transverse momentum, $p_{\perp}>30 \mathrm{GeV}$. We employ PDFs and the strong coupling constant as provided by the NNPDF21LO [41], NNPDF23NLO, and NNPDF23NNLO [42] PDF sets to compute, respectively, LO, NLO, and NNLO cross sections. We set the renormalization and factorization scales to the mass of the Higgs boson

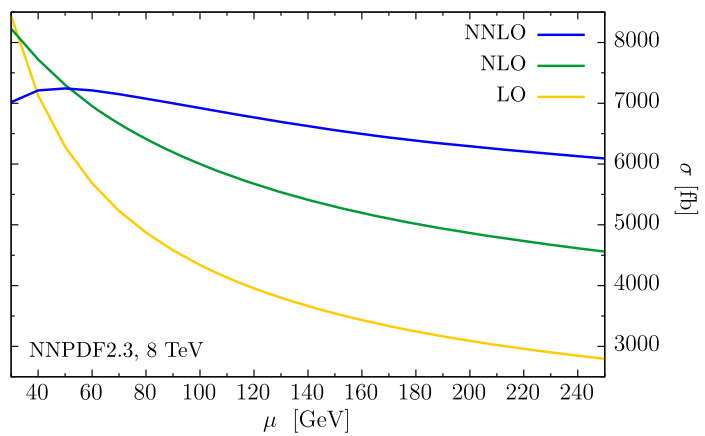

FIG. 2 (color online). Dependence of the total LO, NLO, and NNLO cross sections on the unphysical scale $\mu$. See text for details. 


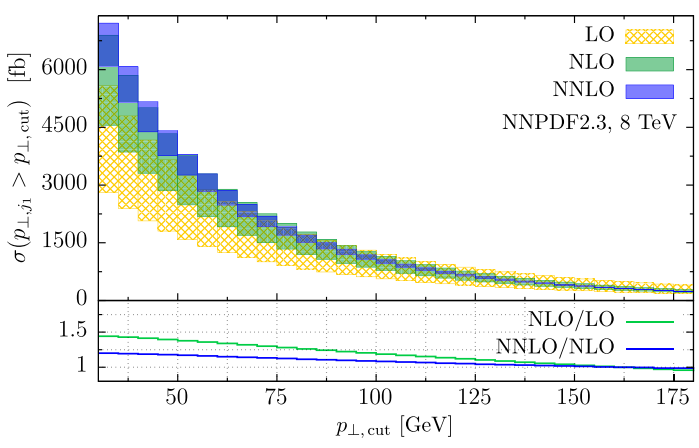

FIG. 3 (color online). Higgs plus jet production cross sections in dependence of the cut on the jet transverse momentum. The minimal cut we consider is $p_{\perp}>30 \mathrm{GeV}$. Color bands show the scale variation uncertainty. The $K$ factor in the lower pane is computed for $\mu=m_{H}$. See text for details.

and we estimate the uncertainty associated with higher orders in perturbation theory by changing the scale by a factor of 2. For the $8 \mathrm{TeV} \mathrm{LHC}$, we find $\sigma_{p p \rightarrow H+j}=$ $3.9_{-1.1}^{+1.7} \mathrm{pb}, \quad 5.6_{-1.1}^{+1.3} \mathrm{pb}, \quad 6.7_{-0.6}^{+0.5} \mathrm{pb}$ at leading, next-toleading, and next-to-next-to-leading order, respectively. Results for $\mu=m_{H} / 2$ and $\mu=2 m_{H}$ are shown as superand subscripts, respectively. For $\mu=m_{H}$, the NLO (NNLO) cross section exceeds the leading order one by $44 \%(72 \%)$, indicating a reasonable convergence of perturbative expansion. The convergence is better for lower scales: for example, for $\mu=m_{H} / 2$ the NLO (NNLO) cross section exceeds the leading order one by $23 \%$ (29\%). As expected, the scale uncertainty is significantly reduced at NNLO. This is also illustrated in Fig. 2, where we plot the total cross section at LO, NLO, and NNLO as a function of the unphysical scale $\mu$ over the range $\mu \in\left[p_{\perp, \text { cut }}: 2 m_{H}\right]$. We estimate the residual uncertainty due to PDF following the PDF4LHC recommendation [43] and find it to be at the $\mathcal{O}(5 \%)$ level. The situation is similar for the $13 \mathrm{TeV}$ LHC. More precisely, we find $\sigma_{p p \rightarrow H+j}=10.2_{-2.6}^{+4.0} \mathrm{pb}, 14.7_{-2.5}^{+3.0} \mathrm{pb}$, $17.5_{-1.4}^{+1.1} \mathrm{pb}$ at leading, next-to-leading, and next-to-next-toleading order, corresponding to a NLO (NNLO) increase with respect to LO of $44 \%$ (72\%) for $\mu=m_{H}$ and of $25 \%$ (31\%) for $\mu=m_{H} / 2$.

It is interesting to understand to what extent perturbative QCD corrections depend on the kinematics of the process and/or on the details of the jet algorithm. One way to study this is to explore how the NNLO QCD corrections change as the lower cut on the jet transverse momentum is varied. We show corresponding results for the $8 \mathrm{TeV}$ LHC in Fig. 3 where the cumulative distribution for $\sigma\left(H+j, p_{\perp, j} \geq\right.$ $\left.p_{\perp, \text { cut }}\right)$ is displayed. The inset in Fig. 3 shows ratios of NNLO(NLO) to $\mathrm{NLO}(\mathrm{LO}) H+j$ cross sections, respectively, computed for $\mu_{F}=\mu_{R}=m_{H}$ as a function of the jet $p_{\perp}$ cut. It follows from Fig. 3 that QCD radiative corrections depend on the kinematics. Indeed, the NNLO to NLO cross sections' ratio changes from 1.25 at $p_{\perp}=$ $30 \mathrm{GeV}$ to $\sim 1$ at $p_{\perp} \sim 150 \mathrm{GeV}$.

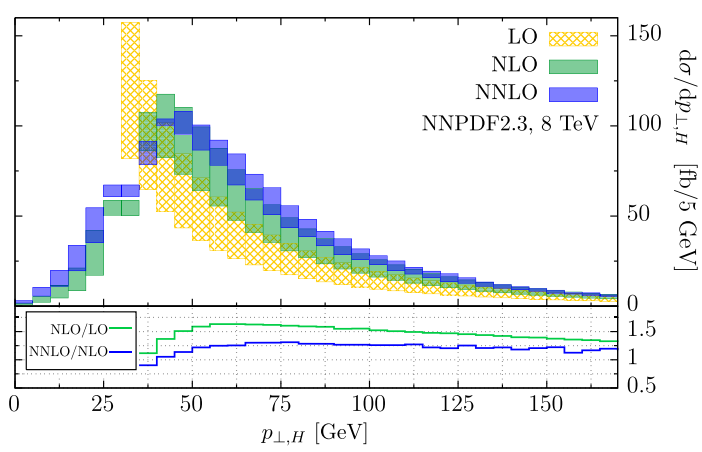

FIG. 4 (color online). Higgs boson transverse momentum distribution in $p p \rightarrow H+j$ at $8 \mathrm{TeV}$ LHC. The jet is defined with the anti- $k_{\perp}$ algorithm with $\Delta R=0.5$ and the cut on the jet transverse momentum of $30 \mathrm{GeV}$. Further details are explained in the text.

In Fig. 4 we show the Higgs boson transverse momentum distribution in the reaction $p p \rightarrow H+j$, for three consecutive orders of perturbation theory. We require that there is a jet in the final state with a transverse momentum higher than $p_{\perp, j}>30 \mathrm{GeV}$. Note that the two bins closest to the boundary $p_{\perp, H}=30 \mathrm{GeV}$ have been combined to avoid the well-known Sudakov-shoulder effect [44]. Away from that region, the NNLO QCD radiative corrections increase the NLO cross section by about $20 \%$, slowly decreasing as $p_{\perp, H}$ increases. Also, we note that the $g g$ and $q g$ channels exhibit a different $p_{\perp}$ behavior, the latter becoming more dominant at higher values of $p_{\perp}$.

In conclusion, we have presented a calculation of the NNLO QCD corrections to the production of the Higgs boson in association with a jet at the LHC. This is the first computation of NNLO QCD corrections to a Higgs production process with a jet in the final state which includes all channels and color factors relevant for LHC phenomenology. It shows that techniques for performing NNLO QCD computations, that were in the development phase for several years, can indeed be used to provide precise predictions for complex processes at hadron colliders. The total cross section for $H+$ jet production receives moderate NNLO QCD corrections. For jets defined with the anti- $k_{\perp}$ algorithm with $p_{\perp, j}>30 \mathrm{GeV}$, we find NNLO QCD corrections of the order of $20 \%$ with respect to NLO for $\mu=m_{H}$. These moderate corrections are the result of the smaller corrections for the $q g$ channel with respect to the $g g$ one, and a suppression of the $g g$ channel due to $q \bar{q}$ final states not considered in previous analyses $[9,10]$. Beyond the total cross section, our computation will have important implications for many processes that are used to study properties of the Higgs boson, including $W^{+} W^{-}$and $\gamma \gamma$ final states, primarily through improved modeling of the Higgs transverse momentum and rapidity distributions. In particular, since the complete nextto-next-to-next-to-leading order computation of the Higgs boson production cross section is available, a consistent 
computation of the $H+0$ jets, $H+1$ jet, $H+2$ jet, and $H+3$ jet exclusive processes becomes possible for the first time. Furthermore, since the Higgs boson is a spin-zero particle, our computation can be easily extended to include Higgs boson decays, to enable theoretical predictions for fiducial cross sections and kinematic distributions for the particles that are observed in detectors. Once this is done, our calculation will provide a powerful tool that will help to understand detailed properties of the Higgs boson at the LHC.

We thank T. Becher, J. Campbell, T. Gehrmann, and M. Jaquier for helpful communications. We are grateful to $\mathrm{S}$. Badger for making his results for tree-level amplitudes available to us. F. C. would like to thank the Institute for Theoretical Particle Physics of KIT and the Physics and Astronomy Department of Northwestern University for hospitality at various stages of this project. R. B. is supported by the DOE under Contract No. DE-AC0206CH11357. F. P. is supported by the DOE Grants No. DEFG02-91ER40684 and No. DE-AC02-06CH11357. This research used resources of the National Energy Research Scientific Computing Center, a DOE Office of Science User Facility supported by the Office of Science of the U.S. Department of Energy under Contract No. DE-AC0205CH11231.

*rboughezal@anl.gov

†fabrizio.caola@cern.ch

*kirill.melnikov@kit.edu

${ }^{\S}$ f-petriello@northwestern.edu

"markus.schulze@cern.ch

[1] G. Aad et al. (ATLAS Collaboration), Observation of a new particle in the search for the standard model Higgs boson with the ATLAS detector at the LHC, Phys. Lett. B 716, 1 (2012).

[2] S. Chatrchyan et al. (CMS Collaboration), Observation of a new boson at a mass of $125 \mathrm{GeV}$ with the CMS experiment at the LHC, Phys. Lett. B 716, 30 (2012).

[3] G. Aad et al. (ATLAS Collaboration), Report No. ATLASCONF-2013-034.

[4] S. Chatrchyan et al. (CMS Collaboration), Report No. CMS-PAS-HIG-13-005.

[5] S. Dawson, A. Gritsan, H. Logan, J. Qian, C. Tully, R. Van Kooten, A. Ajaib, A. Anastassov et al., Working group report: Higgs boson, arXiv:1310.8361.

[6] See, for example, the following study of differential distributions in the $\gamma \gamma$ and $4 l$ channels: G. Aad et al. (ATLAS Collaboration), Measurements of the Total and Differential Higgs Boson Production Cross Sections Combining the $H \rightarrow \gamma \gamma$ and $H \rightarrow Z Z^{*} \rightarrow 4 l$ Decay Channels at $\sqrt{s}=8$ $\mathrm{TeV}$ with the ATLAS Detector, arXiv:1504.05833.

[7] G. Aad et al. (ATLAS Collaboration), Report No. ATLASCONF-2014-009.

[8] C. Anastasiou, C. Duhr, F. Dulat, F. Herzog, and B. Mistlberger, Higgs Boson Gluon-Fusion Production in N3LO QCD, Phys. Rev. Lett. 114, 212001 (2015).
[9] R. Boughezal, F. Caola, K. Melnikov, F. Petriello, and M. Schulze, Higgs boson production in association with a jet at next-to-next-to-leading order in perturbative QCD, J. High Energy Phys. 06 (2013) 072.

[10] X. Chen, T. Gehrmann, E. W. N. Glover, and M. Jaquier, Precise QCD predictions for the production of Higgs + jet final states, Phys. Lett. B 740, 147 (2015).

[11] A. Banfi, G. P. Salam, and G. Zanderighi, NLL+NNLO predictions for jet-veto efficiencies in Higgs-boson and Drell-Yan production, J. High Energy Phys. 06 (2012) 159; A. Banfi, P. F. Monni, G. P. Salam, and G. Zanderighi, Higgs- and Z-boson Production with a Jet Veto, Phys. Rev. Lett. 109, 202001 (2012); X. Liu and F. Petriello, Resummation of jet-veto logarithms in hadronic processes containing jets, Phys. Rev. D 87, 014018 (2013); X. Liu and F. Petriello, Reducing theoretical uncertainties for exclusive Higgs-boson plus one-jet production at the LHC, Phys. Rev. D 87, 094027 (2013); T. Becher, M. Neubert, and L. Rothen, Factorization and $\boldsymbol{N}^{3} \boldsymbol{L} \boldsymbol{L}_{\boldsymbol{p}}+\mathbf{N N L O}$ predictions for the Higgs cross section with a jet veto, J. High Energy Phys. 10 (2013) 125; I. W. Stewart, F. J. Tackmann, J. R. Walsh, and S. Zuberi, Jet $\boldsymbol{p}_{T}$ resummation in Higgs production at NNLL' + NNLO, Phys. Rev. D 89, 054001 (2014); R. Boughezal, X. Liu, F. Petriello, F. J. Tackmann, and J. R. Walsh, Combining resummed Higgs predictions across jet bins, Phys. Rev. D 89, 074044 (2014).

[12] A. Gehrmann-De Ridder, T. Gehrmann, E. W. N. Glover, and J. Pires, Second Order QCD Corrections to Jet Production at Hadron Colliders: The All-Gluon Contribution, Phys. Rev. Lett. 110, 162003 (2013); M. Czakon, P. Fiedler, and A. Mitov, Total Top-Quark Pair-Production Cross Section at Hadron Colliders Through $\boldsymbol{O}\left(\boldsymbol{\alpha}_{S}^{4}\right)$, Phys. Rev. Lett. 110, 252004 (2013); J. Currie, A. GehrmannDe Ridder, E. W. N. Glover, and J. Pires, NNLO QCD corrections to jet production at hadron colliders from gluon scattering, J. High Energy Phys. 01 (2014) 110; M. Brucherseifer, F. Caola, and K. Melnikov, On the NNLO QCD corrections to single-top production at the LHC, Phys. Lett. B 736, 58 (2014); M. Czakon, P. Fiedler, and A. Mitov, Resolving the Tevatron Top Quark Forward-Backward Asymmetry Puzzle, Phys. Rev. Lett. 115, 052001 (2015); R. Boughezal, C. Focke, X. Liu, and F. Petriello, $\boldsymbol{W}$-boson production in association with a jet at next-to-next-toleading order in perturbative QCD, arXiv:1504.02131 [Phys. Rev. Lett. (to be published)].

[13] R. V. Harlander, T. Neumann, K. J. Ozeren, and M. Wiesemann, Top-mass effects in differential Higgs production through gluon fusion at order $\alpha_{s}^{4}$, J. High Energy Phys. 08 (2012) 139.

[14] S. Dawson, I. M. Lewis, and M. Zeng, Effective field theory for Higgs boson plus jet production, Phys. Rev. D 90, 093007 (2014).

[15] K. G. Chetyrkin, B. A. Kniehl, and M. Steinhauser, Decoupling relations to $O\left(\alpha_{s}^{3}\right)$ and their connection to low-energy theorems, Nucl. Phys. B510, 61 (1998).

[16] C. Anastasiou and K. Melnikov, Higgs boson production at hadron colliders in NNLO QCD, Nucl. Phys. B646, 220 (2002).

[17] M. Cacciari, G. P. Salam, and G. Soyez, The anti- $k_{t}$ jet clustering algorithm, J. High Energy Phys. 04 (2008) 063. 
[18] S. Buehler and A. Lazopoulos, Scale dependence and collinear subtraction terms for Higgs production in gluon fusion at N3LO, J. High Energy Phys. 10 (2013) 096.

[19] J. M. Campbell and R. K. Ellis, MCFM for the Tevatron and the LHC, Nucl. Phys. B, Proc. Suppl. 205-206, 10 (2010).

[20] T. Gehrmann, M. Jaquier, E. W. N. Glover, and A. Koukoutsakis, Two-loop QCD corrections to the helicity amplitudes for $H \rightarrow 3$ partons, J. High Energy Phys. 02 (2012) 056.

[21] C. F. Berger, V. Del Duca, and L. J. Dixon, Recursive construction of Higgs+multiparton loop amplitudes: The last of the " $\Phi$-nite" loop amplitudes, Phys. Rev. D 74, 094021 (2006); 76, 099901(E) (2007); S. D. Badger and E. W. N. Glover, One-loop helicity amplitudes for $\boldsymbol{H} \rightarrow$ gluons: The all-minus configuration, Nucl. Phys. B, Proc. Suppl. 160, 71 (2006); S. D. Badger, E. W. N. Glover, and $\mathrm{K}$. Risager, One-loop $\Phi$-MHV amplitudes using the unitarity bootstrap, J. High Energy Phys. 07 (2007) 066; E. W. N. Glover, P. Mastrolia, and C. Williams, One-loop $\Phi-$ MHV amplitudes using the unitarity bootstrap: The general helicity case, J. High Energy Phys. 08 (2008) 017; S. D. Badger and E. W. N. Glover, P. Mastrolia, and C. Williams, One-loop Higgs plus four gluon amplitudes: Full analytic results, J. High Energy Phys. 01 (2010) 036; L. J. Dixon and Y. Sofianatos, Analytic one-loop amplitudes for a Higgs boson plus four partons, J. High Energy Phys. 08 (2009) 058; S. Badger, J. M. Campbell, R. K. Ellis, and C. Williams, Analytic results for the one-loop NMHV Hqqqgg amplitude, J. High Energy Phys. 12 (2009) 035.

[22] S. D. Badger (to be published).

[23] M. Czakon, A novel subtraction scheme for double-real radiation at NNLO, Phys. Lett. B 693, 259 (2010).

[24] M. Czakon, Double-real radiation in hadronic top quark pair production as a proof of a certain concept, Nucl. Phys. B849, 250 (2011).

[25] R. Boughezal, K. Melnikov, and F. Petriello, A subtraction scheme for NNLO computations, Phys. Rev. D 85, 034025 (2012).

[26] M. Czakon and D. Heymes, Four-dimensional formulation of the sector-improved residue subtraction scheme, Nucl. Phys. B890, 152 (2015).

[27] T. Binoth and G. Heinrich, Numerical evaluation of multiloop integrals by sector decomposition, Nucl. Phys. B680, 375 (2004).

[28] C. Anastasiou, K. Melnikov, and F. Petriello, A new method for real radiation at NNLO, Phys. Rev. D 69, 076010 (2004).
[29] T. Binoth and G. Heinrich, Numerical evaluation of phase space integrals by sector decomposition, Nucl. Phys. B693, 134 (2004).

[30] S. Frixione, Z. Kunszt, and A. Signer, Three jet cross-sections to next-to-leading order, Nucl. Phys. B467, 399 (1996).

[31] S. Catani, D. de Florian, and M. Grazzini, Universality of non-leading logarithmic contributions in transversemomentum distributions, Nucl. Phys. B596, 299 (2001).

[32] F. A. Berends and W. T. Giele, , Multiple soft gluon radiation in parton processes, Nucl. Phys. B313, 595 (1989).

[33] J. M. Campbell and E. W. N. Glover, Double unresolved approximations to multiparton scattering amplitudes, Nucl. Phys. B527, 264 (1998).

[34] S. Catani and M. Grazzini, Collinear factorization and splitting functions for next-to-next-to-leading order QCD calculations, Phys. Lett. B 446, 143 (1999).

[35] Z. Bern, V. Del Duca, W. B. Kilgore, and C. R. Schmidt, Infrared behavior of one-loop QCD amplitudes at next-tonext-to-leading order, Phys. Rev. D 60, 116001 (1999).

[36] S. Catani and M. Grazzini, Infrared factorization of treelevel QCD amplitudes at the next-to-next-to-leading order and beyond, Nucl. Phys. B570, 287 (2000).

[37] S. Catani and M. Grazzini, The soft-gluon current at oneloop order, Nucl. Phys. B591, 435 (2000).

[38] D. A. Kosower and P. Uwer, One loop splitting amplitudes in gauge theory, Nucl. Phys. B563, 477 (1999).

[39] J. M. Campbell, R. K. Ellis, and G. Zanderighi, Next-toleading order Higgs +2 jet production via gluon fusion, J. High Energy Phys. 10 (2006) 028.

[40] R. Boughezal, C. Focke, W. Giele, X. Liu, and F. Petriello, Higgs boson production in association with a jet using jettiness subtraction, Phys. Lett. B 748, 5 (2015).

[41] R. D. Ball, V. Bertone, F. Cerutti, L. Del Debbio, S. Forte, A. Guffanti, J. I. Latorre, J. Rojo, and M. Ubiali, Impact of heavy quark masses on parton distributions and LHC phenomenology, Nucl. Phys. B849, 296 (2011).

[42] R. D. Ball, V. Bertone, S. Carrazza, C. S. Deans, L. Del Debbio, S. Forte, A. Guffanti, N. P. Hartland et al., Parton distributions with LHC data, Nucl. Phys. B867, 244 (2013).

[43] M. Botje, J. Butterworth, A. Cooper-Sarkar, A. de Roeck, J. Feltesse, S. Forte, A. Glazov J. Huston et al., The PDF4LHC working group interim recommendations, arXiv: 1101.0538.

[44] S. Catani and B. R. Webber, Infrared safe but infinite: Soft gluon divergences inside the physical region, J. High Energy Phys. 10 (1997) 005. 